\title{
AUTOIMMUNE THYROIDITIS AMONG IONIZING RADIATION EXPOSED WORKERS IN CARDIAC CATHETERIZATION UNITS.
}

\author{
By \\ ${ }^{1}$ Farahat SA, ${ }^{1}$ Mansour N, ${ }^{2}$ Sheta MM, ${ }^{3}$ Alramlawy SA and ${ }^{1}$ Ramadan M \\ ${ }^{1}$ Department of Occupational and Environmental Medicine, ${ }^{2}$ Department of Clinical pathology, \\ ${ }^{3}$ Department of Critical Care, Faculty of Medicine, Cairo University
}

\begin{abstract}
Introduction: It is well known that ionizing radiation (IR) exposure increases the risk of thyroid cancer; however less is known about its role in development of autoimmune thyroiditis. Aim of work: to investigate the thyroid gland functions, level of anti TPO among interventional cardiology team members searching for an association between the level of their occupational exposure to ionizing radiation and the thyroid gland integrity. Materials and methods: This work was carried out in Cardiac Catheterization Unit in National Heart Institute Center. The study comprised two groups, an exposed group of 47 cardiac catheterization workers (19 physicians, 15 nurses and 13 technicians) and a control group of 47 workers matched with the exposed group as regards age and sex. The studied groups were subjected to a detailed questionnaire, full clinical examinations. laboratory investigations in the form of serum thyroid stimulating hormone (TSH), Triiodothyronine (T3), Thyroxine (T4) and antithyroxiperoxidase antibodies (anti-TPO). Radiation exposure was monitored by digital dosimeter. Results: Dosimeter readings were higher among physicians followed by nurses and technicians. Statistically significant higher level of serum TSH, anti-TPO along with statistically significant lower levels of T3, were found among the exposed compared to control groups $(\mathrm{P}<0.05)$. Significant positive correlation was detected between the level of anti-TPO and TSH $(\mathrm{r}=0.876 \mathrm{P}<0.001)$. Moreover, a significant negative correlation was found between anti-TPO and each of T3 $(r=-0.814 \mathrm{P}<0.001)$ and T4 $(r=-0.324 \mathrm{P}<0.05)$. ANOVA and Post Hoc tests showed significantly higher level of anti-TPO with significantly lower levels of T3 among the physicians compared to control group. Conclusion: Autoimmune thyroiditis may be considered as one of the adverse health hazards of occupational exposure to ionizing radiation in cardiac catheterization units. Recommendations: We recommend the regular measurement of thyroid hormone especially TSH and anti-TPO to detect early affection of thyroid gland among radiation exposed workers.
\end{abstract}

Key words: Ionizing radiation, Autoimmune thyroiditis, T3, T4, TSH and Anti-TPO. 


\section{Introduction}

Medical exposure to ionizing radiation from $\mathrm{X}$ - rays and in nuclear medicine is known to be the largest man made source of radiation exposure (Mettler et al., 2009). Such exposure is of considerable concern for interventional cardiologists due to increasing workloads and intricacy of procedures over the last decade (Cousins et al., 2013). Moreover, interventional cardiologists encounter much more radiation exposure than most other medical staff due to their working position near to the X-ray beam and the patient (the source of scatter radiation) (Rehani and Ortiz-Lopez, 2006).

Radiation exposure can exert a number of adverse effects on many tissues in the body. One of the target organs for radiation-related damage is the thyroid gland as it receives a considerable radiation dose from scatter radiation probably due to its anatomical site (Eheman et al., 2003).

While the association between thyroid irradiation and an increased risk of thyroid neoplasia is well established, less attention has been devoted to the potential effects of radiation on the function of the thyroid gland. However, since the Chernobyl accident, some studies have linked radiation exposure to an increased risk of autoimmune thyroid disease (AITD) (Bufalo et al., 2011, Hamatani et al., 2008). On the other hand, earlier studies could not confirm such association (Yoshimoto et al., 1995).

The external radiation may expose thyroidal antigens to the immune system and thus induce autoimmunity by stimulation of dendritic cells (Goodnow, 2001) or may cause direct destruction of the thyroid gland (Agate et al., 2008). However, the mechanisms and the pathways involved in that relation remain not well understood and should be investigated (Ostroumova et al., 2009).

Autoimmune thyroid disease is the most common organ-specific autoimmune disease and characterized by the presence auto-antibodies directed against several major thyroid antigen. Among those, are the Anti-TPO autoantibodies (anti TPO Ab) which were found in over $90 \%$ of patients with autoimmune hypothyroidism and 70\% of patient with Graves' disease (Hawa et al., 2006). Moreover, a dose-response relation between radiation exposure 
and the prevalence of positive antithyroxiperoxidase antibodies was found in some studies (Völzke et al., 2005).

Ionizing radiation can produce functional alteration of the immune system and breaks self -tolerance with induction of autoimmune disease among that is the thyroid gland which is one of the susceptible organs for the deleterious effect of ionizing radiation. However, the association and pathogenesis of radiation induced autoimmune thyroiditis is still not yet clear (Ostroumova et al., 2009).

\section{Aim of work}

This study was carried out to investigate the thyroid gland functions, level of anti TPO among interventional cardiology team members searching for an association between the level of their occupational exposure to ionizing radiation and the thyroid gland integrity.

\section{Materials and methods}

- Study design: A cross sectional study

- Place and duration of study: the study was carried out in the National Heart Institute Center during the period from March 2014 to April 2015.
- Study sample: All eligible personnel were invited to participate in the study.

Inclusion criteria for ionizing radiation exposed subjects included regular employment in the catheterization department for at least the preceding 5 years.

Exclusion criteria included: those having diseases affecting hypothalamic pituitary axis, surgical intervention to thyroid gland, intake of immunosuppressive drugs or receiving radiotherapy. Those who met the inclusion criteria and agreed to participate were 47 subjects ( 31 males \& 16 females). For the purpose of the study, exposed group was further subdivided into19 cardiologists, 15 nurses and 13 technicians.

A control group of 47 individuals were randomly selected as to be matched with the exposed population as regards age, gender and special habits of medical important. They were selected from clinical pathology department with no history of exposure to ionizing radiation, diseases affecting hypothalamic pituitary axis, surgical intervention to thyroid gland, intake of immunosuppressive drugs or receiving radiotherapy. The 
control group consisted of 14 physicians, 18 nurses, 15 technicians.

\section{Study methods:}

\section{-Radiation exposure assessment}

The radiation exposure of cardiac catheterization team members was assessed using a digital dosimeter in both procedures (diagnostic and therapeutic) over three months. The effective dose $(\mu \mathrm{Sv})$ received was determined according to the following formula:

Effective dose $=($ dose of procedure - background radiation) $\times$ conversion factor.

The background radiation was determined by the formula:

Procedure time (in seconds) $\times$ $0.00004 \mu \mathrm{Sv} / \mathrm{s}$, considering a conversion factor of 1.01(Cardiso et al., 2014)

The dosimeter was positioned over the lead apron and reseted at the beginning of the procedure, and the final dose was measured at the end.

Three dosimeters were used: one for the operating physician conducting the procedure, one for the nurse and the other for the technician who assisted the exam. All members used radiological protection equipment (an apron) but limited number used the thyroid shield.

\section{-Questionnaire and clinical examination}

Every subject in the study was subjected to a specially designed detailed questionnaire including medical history, personal, present, past, family, occupational, menstrual and obstetric history. General and local systemic examinations were carried out with special attention to acute and chronic long term effect of radiation. Thyroid gland examination was performed using inspection and palpation.

\section{-Laboratory investigations}

A blood sample of $5 \mathrm{ml}$ was drawn from each subject through venipuncture from the arm using a dry plastic syringe and allowed to clot then centrifuged for separation of the serum for determination of thyroid hormone levels (TSH, T3, and T4) and anti-thyroxiperoxidase antibody.

\section{A. Thyroid function profile (TSH,} free $T 3$, free $T 4$ )

The levels of these hormones were measured by electrochemiluminescence immunoassay (ECLIA). 
The normal reference ranges are taken as: $0.4-4 \mathrm{uI} / \mathrm{mL}$ for TSH, 2-4.4 pg/ $\mathrm{mL}$ for FT3 and 0.9-1.8ng/dL for FT4

\section{B. Anti-thyroxiperoxidase antibody} (anti-TPO):

Using Enzyme-linked immunosorbent assay method for the quantitative determination of specific $\operatorname{IgG}$ autoantibodies to TPO in human serum done by the AutostatTMII assay kits for detection of autoantibodies is a solid phase immunosorbent assay (ELISA) in which the analyte is indicated by a colour reaction of an enzyme and substrate.

Interpretation of results of Anti TPO $\mathrm{U} / \mathrm{ml}$

$<35$ negative $35-50$ equivocal $>50$ positive

\section{Consent}

Prior to this study, approval of the cardiac catheterization department was obtained. An oral consent for sharing in the study was voluntarily obtained from each individual after explanation of the aim of the study. Strict confidentiality was carried out through all the study.

\section{Ethical approval}

The study protocol was approved by the ethical committee of the Department of Occupational and Environmental Medicine, Faculty of medicine, Cairo University.

\section{Data management}

Data were analyzed using SPSS 15. The mean values, standard deviation (SD), median and ranges were then estimated for quantitative variables, as for the qualitative variables, the frequency distribution was calculated.

Comparisons between exposed and control groups were done using Chi Square $\left(\mathrm{X}^{2}\right)$ test for qualitative variables and using the independent simple t-test for normally distributed quantitative variable as well as the analysis of variance (ANOVA test) after normality of all Quantitative variables. The nonparametrical Mann-Whitney (Z-test) was used for quantitative variables not normally distributed. A correlation (r) was done to test for the presence of linear relations between quantitative variables. P-values less than 0.05 and less than 0.001 were considered statistically significant and highly significant, respectively. 


\section{Results}

This study was carried out on the cardiac catheterization team members exposing to ionizing radiation working in National Heart Institute Center during the period from March 2014 to April 2015.The study population consisted of 47 exposed workers comprising 31 males and 16 females with the mean age (34.17 \pm 2.98$)$ and duration of work
(9.32 \pm 4.32$)$. Another 47 individuals (control group) comprising 27 males and 20 females were selected as to be matched with the exposed population with the mean age and duration of work (34.27 \pm 2.97 and 10.8 \pm 3.6 respectively)

Comparing the subject demographic data of both groups revealed that there was no significance difference between both groups $(\mathrm{p}>0.05)$.

Table (1): The frequency distribution of thyroid manifestation among the exposed and control groups:

\begin{tabular}{|l|c|c|c|c|c|c|}
\hline \multirow{2}{*}{ Thyroid manifestations } & \multicolumn{2}{|c|}{$\begin{array}{c}\text { Exposed } \\
\mathbf{n = 4 7}\end{array}$} & \multicolumn{2}{c|}{$\begin{array}{c}\text { control } \\
\mathbf{n = 4 7}\end{array}$} & $\mathbf{X}^{\mathbf{2}}$ & p value \\
\cline { 2 - 7 } & $\mathbf{N o}$ & $\mathbf{\%}$ & $\mathbf{N o}$ & $\mathbf{\%}$ & & \\
\hline Manifestations of hypothyroidisim & & & & & & \\
Recent weight gain & 5 & 10.6 & 2 & 4.3 & 1.38 & $>0.05$ \\
Intolerance to cold & 4 & 8.5 & 1 & 2.1 & 1.9 & $>0.05$ \\
Easy fatigability & 19 & 40.4 & 3 & 6.4 & 15.19 & $<0.05^{*}$ \\
\hline Manifestations of hyperthyroidism & & & & & & \\
Weight loss & 2 & 4.3 & 1 & 2.1 & 0.34 & $>0.05$ \\
Anxiety & 5 & 10.6 & 3 & 6.4 & 0.54 & $>0.05$ \\
Tremors & 2 & 4.3 & 0 & 0 & 2.04 & $>0.05$ \\
\hline Thyroid examination & & & & & & \\
Palpable thyroid & 2 & 4.3 & 0 & 0 & 2.04 & $>0.05$ \\
Thyroid nodules & 1 & 2.1 & 0 & 0 & 1.01 & $>0.05$ \\
\hline
\end{tabular}

*: Statistically significant.

Table (1): shows that there is no statistical difference as regard thyroid manifestation among both exposed and control groups apart from easy fatigability which was statistically significant higher among exposed group compared to control.

Other symptoms and signs of hyperthyroidism and hypothyroidism were not evident in both exposed and control group like (constipation, hair loss, polyphagia, insomnia , intolerance to hot weather and skin changes). 
Table (2): Mean \pm SD and range of the thyroid function parameters (TSH, T3, T4) and anti-thyroxiperoxidase antibodies (TPO) among exposed and control subjects.

\begin{tabular}{|c|c|c|c|c|c|c|}
\hline & $\begin{array}{c}\text { Exposed } \\
n=47\end{array}$ & $\begin{array}{c}\text { Control } \\
\mathrm{n}=47\end{array}$ & $\mathbf{Z}$ & $p$ value & $X^{2}$ & $p$ value \\
\hline \begin{tabular}{|l|} 
Anti TPO \\
$(50 \mathrm{u} / \mathrm{ml})$
\end{tabular} & $\begin{array}{c}55.23 \pm 69.65 \\
(4.3-399.5) \\
\end{array}$ & $\begin{array}{l}18.7 \pm 19.95 \\
(3.3-100.4) \\
\end{array}$ & 3.108 & $<0.05^{*}$ & & \\
\hline $\begin{array}{l}\text { TSH } \\
(0.4-4 \mu \mathrm{IU} / \mathrm{ml})\end{array}$ & $\begin{array}{c}2.26 \pm 1.02 \\
(0.5-4.9)\end{array}$ & $\begin{array}{c}1.86 \pm 0.707 \\
(0.4-3.5)\end{array}$ & 2.215 & $<0.05^{*}$ & $\begin{array}{c}1.38 \\
1.9 \\
15.19\end{array}$ & $\begin{array}{l}>0.05 \\
>0.05 \\
>0.05^{*}\end{array}$ \\
\hline $\begin{array}{l}\mathrm{T} 4 \\
(0.9-1.8 \mathrm{ng} / \mathrm{ml})\end{array}$ & $\begin{array}{c}1.425 \pm 0.247 \\
(0.9-1.9)\end{array}$ & $\begin{array}{c}1.49 \pm 0.21 \\
(1.1-1.9)\end{array}$ & $1.315^{\#}$ & $>0.05$ & $\begin{array}{l}0.34 \\
0.54 \\
2.04\end{array}$ & $\begin{array}{l}<0.05 \\
>0.05 \\
<0.05\end{array}$ \\
\hline $\begin{array}{l}\mathrm{T3} \\
(2-4.4 \mathrm{pg} / \mathrm{ml})\end{array}$ & $\begin{array}{c}2.69 \pm 0.58 \\
(1.6-3.8)\end{array}$ & $\begin{array}{c}3.17 \pm 0.34 \\
(2.5-3.9)\end{array}$ & $4.839^{\#}$ & $<0.001^{*}$ & $\begin{array}{l}2.04 \\
1.01\end{array}$ & $\begin{array}{l}>0.05 \\
<0.05\end{array}$ \\
\hline
\end{tabular}

\# Student's t test

*: Statistically significant

Table (2) shows that the mean levels of TSH and anti-TPO were statistically significantly higher, and the mean level of T3 was statistically significantly lower among the exposed group compared to their referent control. No statistically significant difference was detected between both studied groups regarding T4.

By analyzing the individual levels for anti-TPO and thyroid hormones (TSH, T3, and T4), the entire exposed group had normal thyroid function and 17 exposed personals had positive anti-TPO, as level exceeding $50 \mathrm{U} / \mathrm{ml}$. 
Table (3): Correlation coefficient between anti TPO on one hand and TSH, T3 and T4 on the other hand among exposed group.

\begin{tabular}{|c|c|c|c|c|c|c|}
\hline \multicolumn{3}{|c|}{ anti-TPO } & \multicolumn{2}{|c|}{$\begin{array}{c}\text { control } \\
n=47\end{array}$} & \multirow[t]{2}{*}{$\mathbf{X}^{2}$} & \multirow[t]{2}{*}{$p$ value } \\
\hline & $\mathrm{R}$ & $\mathrm{p}$ value & No & $\%$ & & \\
\hline $\begin{array}{l}\text { TSH } \\
(\mu \mathrm{Iu} / \mathrm{ml})\end{array}$ & 0.876 & $<0.001 *$ & $\begin{array}{l}2 \\
1 \\
3\end{array}$ & $\begin{array}{l}4.3 \\
2.1 \\
6.4\end{array}$ & $\begin{array}{c}1.38 \\
1.9 \\
15.19\end{array}$ & $\begin{array}{l}>0.05 \\
>0.05 \\
<0.05^{*}\end{array}$ \\
\hline $\begin{array}{l}\text { T3 } \\
(\mathbf{p g} / \mathrm{ml})\end{array}$ & -0.814 & $<0.001^{*}$ & $\begin{array}{l}1 \\
3 \\
0\end{array}$ & $\begin{array}{c}2.1 \\
6.4 \\
0\end{array}$ & $\begin{array}{l}0.34 \\
0.54 \\
2.04\end{array}$ & $\begin{array}{l}>0.05 \\
>0.05 \\
>0.05\end{array}$ \\
\hline $\begin{array}{l}\text { T4 } \\
\text { (ng/dl) }\end{array}$ & -0.324 & $<0.05^{*}$ & $\begin{array}{l}0 \\
0\end{array}$ & $\begin{array}{l}0 \\
0\end{array}$ & $\begin{array}{l}2.04 \\
1.01\end{array}$ & $\begin{array}{l}>0.05 \\
>0.05\end{array}$ \\
\hline
\end{tabular}

*: Statistically significant

Table (3) shows that there were statistically significant positive correlations between anti-TPO level and TSH. On the other hand significant negative correlations were detected between anti-TPO and each of T3 and T4. 
Table (4): ANOVA of mean \pm SD of thyroid function (TSH,T3 and T4) and anti TPO among different categories of the studied groups.

\begin{tabular}{|l|c|c|c|c|c|c|}
\hline & $\begin{array}{c}\text { Physicians } \\
\mathbf{n = 1 9}\end{array}$ & $\begin{array}{c}\text { Nurses } \\
\mathbf{n = 1 5}\end{array}$ & $\begin{array}{c}\text { Technicians } \\
\mathbf{n = 1 3}\end{array}$ & $\begin{array}{c}\text { Control } \\
\mathbf{n = 4 7}\end{array}$ & F test & $\mathbf{p}$ value \\
\hline $\begin{array}{l}\text { anti TPO } \\
(\mathbf{U} / \mathbf{m l})\end{array}$ & $70.45 \pm 91.8$ & $45.92 \pm 58$ & $33.31 \pm 33.7$ & $18.7 \pm 19.95$ & 5.459 & $<0.05^{*}$ \\
\hline $\begin{array}{l}\text { TSH } \\
(\boldsymbol{\mu I U} / \mathbf{m l})\end{array}$ & $2.32 \pm 0.9$ & $2.4 \pm 1.2$ & $1.99 \pm 0.99$ & $1.88 \pm 0.7$ & 2.243 & $>0.05$ \\
\hline $\begin{array}{l}\text { T3 } \\
(\mathbf{p g} / \mathbf{m l})\end{array}$ & $2.71 \pm 0.545$ & $2.67 \pm 0.52$ & $2.76 \pm 0.73$ & $3.17 \pm 0.337$ & 7.65 & $<0.001^{*}$ \\
\hline $\begin{array}{l}\mathbf{T 4} \\
(\mathbf{n g} / \mathbf{m l})\end{array}$ & $1.45 \pm 0.24$ & $1.433 \pm 0.25$ & $1.37 \pm 0.248$ & $1.48 \pm 0.21$ & 0.859 & $>0.05$ \\
\hline
\end{tabular}

*: Statistically significant

Table (4) shows that there is a statistically significant difference regarding antiTPO and T3 among the different job categories of the studied groups.

By analyzing individual doses for anti-TPO among physicians, nurses and technicians, there was positive anti-TPO level exceeding $50 \mathrm{U} / \mathrm{ml}$ in 8 physicians with range $(63-399.5 \mathrm{U} / \mathrm{ml}), 6$ nurses with range $(68-200.6 \mathrm{U} / \mathrm{ml})$ and 3 technicians with range $(68-97.6 \mathrm{U} / \mathrm{ml})$.

Table (5): The Effective dose for both diagnosis and therapeutic cardiac procedures among different study categories.

\begin{tabular}{|l|c|c|c|}
\hline & $\begin{array}{c}\text { Diagnostic cardiac } \\
\text { procedure } \\
\text { Range }(\boldsymbol{\mu S v})\end{array}$ & $\begin{array}{c}\text { Therapeutic cardiac } \\
\text { procedure } \\
\text { Range }(\boldsymbol{\mu S v})\end{array}$ & $\begin{array}{c}\text { Technicians } \\
\mathbf{n}=\mathbf{1 3}\end{array}$ \\
\hline Physicians & $15-22.7$ & $35.2-56.9$ & $33.31 \pm 33.7$ \\
\hline Nurses & $12-20.6$ & $30.5-51.6$ & $1.99 \pm 0.99$ \\
\hline Technicians & $1-1.8$ & $2.1-4.66$ & $2.76 \pm 0.73$ \\
\hline
\end{tabular}

Table (5) shows that physicians are the most exposed to ionizing radiation followed by nurses and technicians. 


\section{Discussion}

Our results showed no statistically significant difference between exposed and control group as regards the frequency of symptoms of hypo and hyperthyroidism out of high frequency of easy fatigability among exposed group . However, local thyroid examination revealed two exposed personnel with palpable thyroid gland. Further ultrasonic examination showed the presence of nodular thyroid pattern in the first case and diffuse enlargement in the other. Similar echogenic pattern was detected by Nizovtsiova and Mozhzhukhina, 2005, who found thyroid changes by ultrasound examination among $22.2 \%$ x-ray physicians which appeared mainly as nodular masses. Recently, Elzaki et al., 2012, surveyed the development of thyroid nodules among radiographers and concluded that the prolonged lowdose exposure to ionizing radiation may increase in the risk of thyroid nodules.

Basic parameters of thyroid function (TSH, T3, T4) were within normal range among individuals of exposed and control groups. However, evaluation of thyroid function among our exposed group compared to the control revealed a significant increase in the mean level of TSH with reduction of mean T3 and T4. This reduction reached the significant level only for $\mathrm{T} 3$ $(\mathrm{P}<0.05)$. It is worth noting that TSH is a better indicator for early thyroid failure than T4. This means that TSH may be affected early without changes in T4, a situation known as subclinical hypothyroidism or hyperthyroidism (Biondi and Cooper, 2008). In the current study the high level of TSH, although it is still within the normal range, shows the tendency of the exposed individuals to develop hypothyroidisim. This tendency was clearly demonstrated on further subdivision of the exposed group. TSH level was higher among physicians and nurses than technicians $(2.32 \pm 0.9,2.4 \pm 1.2,1.99 \pm 0.99 \mu \mathrm{IU} / \mathrm{ml}$ respectively) revealing that physicians and nurses are more susceptible to the occurrence of hypothyroidism.

Many studies have concluded that exposure to ionizing radiation is related to the risk of AITDs which involve both hyperthyroidism (Grave's disease) and hypothyroidism (Hashimoto thyroiditis). Nevertheless, all exposed 
personnel in the current study displayed tendency for hypothyroidism. This can be explained by evidences reported by Ron and Brenner, (2010), when they stated that there is an evidence that thyroid irradiation at high doses $(\geq 15$ Gy) could be associated with increased risk of hyperthyroidism, however the magnitude and shape of the dose response remain uncertain. The data concerning risk of hyperthyroidism following medium to low thyroid doses of irradiation are particularly sparse. On the other hand, thyroid irradiation at high, mediate and low dose could be associated with increased risk of hypothyroidism.

Our results were in accordance with Kindler et al., (2006), who studied thyroid disorders in employees of nuclear power plant and reported that occupational exposure to ionizing radiation within the upper allowed dose range was associated with an increased risk of elevated serum TSH. Furthermore, Abd Oun et al., (2013), examined Iraqi cleanup workers at Al-Tuwaitha nuclear Research site for the prevalence of thyroid disorders. They showed that there were two cases associated with clinically observed hypothyroidism. The other workers showed hormonal disturbance results suggesting the presence of subclinical hypothyroidism.

Various thyroid auto-antibodies including thyroid stimulating antibody, anti- thyroglobulin antibody and anti-thyroxiperoxidase antibody are detectable in autoimmune thyroid diseases. The latter is being a sensitive tool for the detection of early subclinical autoimmune thyroid diseases; follow up of the response to immunotherapy and identification of at-risk cases for autoimmune thyroid diseases (Kemp, 2004). Thyroid peroxidase is responsible for the iodination of tyrosine residues in the thyroglobulin molecule. Anti- TPO antibody has been shown to mediate thyroid cell destruction in vitro. Elevated levels of anti-TPO are seen in more than $90 \%$ cases of Hashimoto thyroiditis and about $75 \%$ of Graves» disease cases (Ai et al., 2003).

In the current study estimation of anti-TPO level as a marker for autoimmune thyroiditis revealed a positive anti-TPO among 17 exposed subjects (level above 50U/ml), 8 of 
them were doctors with range (63$399.5 \mathrm{U} / \mathrm{ml}), 6$ nurses $(68-200.6 \mathrm{U} / \mathrm{ml})$ and 3 technicians $(68-97.6 \mathrm{U} / \mathrm{ml})$. Also, its correlation with clinical findings revealed that the first case with nodular thyroid showed normal level of anti-TPO while the other case showed a very high level of anti-TPO (394U/ml). Further, a statistically significant increase in antiTPO level was found among exposed group compared to control $(\mathrm{P}<0.05)$. It is worth noting that its appearance in the circulation may occur several years before clinical manifestation of autoimmune thyroiditis (Walsh et al., 2005 and Balazs, 2012). In the current study the elevated level of anti-TPO was associated with normal levels of thyroid hormones which show the tendency for future development of autoimmune hypothyroidism. This was clearly demonstrated on further subdivision of exposed group. Anti-TPO level was higher among physicians and nurses than technicians $(70.45 \pm 91.8,45.92 \pm 58$ and $33.31 \pm 33.7$ respectively) again revealing that physicians and nurses are more susceptible to the occurrence of autoimmune hypothyroidism.
Furthermore, the increase of antiTPO was in significantly positive correlation with TSH level $(r=0.876$ $\mathrm{p}<0.001)$. On the other hand, the high anti-TPO was significantly in negative correlation with each $\mathrm{T} 3(\mathrm{r}=-0.814$ $\mathrm{p}<0.001)$ and $\mathrm{T} 4(\mathrm{r}=-0.324 \mathrm{p}<0.05)$.

Our results were in accordance with Völzke et al., (2005), who studied the association between occupational exposures to ionizing radiation with autoimmune thyroid disease. They postulated that occupational exposure to ionizing radiation is associated with a dose dependent risk of autoimmune thyroidits. Also, assessment of the effects of childhood radiation exposure on thyroid auto-antibodies and thyroid function 13-15 yrs after the Chernobyl accident revealed that radioactive fallout elicited a transient autoimmune reaction, without triggering full-blown thyroid autoimmune disease (increase level of anti-thyroxiperoxidase antibodies without changes in thyroid hormones) (Agate et al., 2008).

Ghoraishian et al., (2006) as well, found that there is significant correlation between high serum antiTPO and both elevated TSH level and 
decrease in T4 level $(\mathrm{r}=0.107,-0.160$ respectively). They postulated that antiTPO is a good marker for individuals with autoimmune hypothyroidism who have high levels of TSH or less than normal T3 or T4. However, Al-Juburi et al., (2015), reported that there was no relationship between either of serum T3, T4 or TSH concentration and antiTPO level.

Another aspect of the study was to assess radiation dose received by the members of interventional cardiac catheterization team which can vary by many factors including the type of procedure and given patient dose (Kim et al., 2008). In this work, we tried to calculate the radiation dose received by each of physicians, nurses and technicians during both diagnostic and therapeutic cardiac procedures using digital dosimeter. The readings were higher in therapeutic procedures in the order of physicians (range 35.2-56.9 $\mu \mathrm{Sv})$ followed by nurses (31.5-51.6 $\mu \mathrm{Sv}$ ) while technicians had the lowest readings (2.1-4.66 $\mu \mathrm{Sv})$. This difference may be attributed to variable daily working hours and nature of duties. A similar pattern of measurements was reported by Chida et al., (2013), who studied the annual occupational dose for interventional radiology staff; physicians, nurses and technicians. They showed that the annual occupational dose was in order physician $>$ nurses $>$ technicians. However, the radiology staff in the later study was wearing two dosimeters with strict adherence to radiation protection measures.

\section{Conclusion}

Interventional cardiology team especially physicians and nurses were exposed to high dose of ionizing radiation which may carry the risk for development of autoimmune hypothyroidism.

\section{Recommendations}

Generally, the greatest source of radiation exposure to the operator and staff in cardiac catheterization units is the scatter from the patient. Therefore, the current study recommends the following:-

Proper controlling of the patient dose, minimizing the fluoroscopy time, proper shielding of the catheterization room, availability of transparent ceilingsuspended shields and table-suspended 
drapes (hang from the side of the patient table, between the under-table $\mathrm{x}$-ray and the operator) all these should always be employed, as they have been shown to substantially reduce operator dose a long with regular use of thyroid shield.

Biological monitoring of Thyroid hormones level especially TSH and Anti-TPO level to detect early affection of the thyroid gland among radiation exposed workers.

\section{Conflict of interest}

Authors have declared that no conflict of interests exists.

\section{References}

1. Abd Oun M, AL-Thwani A, Khudaier S and Kamel L (2013): Thyroid Examination in LowRadiation Exposed Iraqi Clean-up Workers Immunogenetic Study. Journal of US-China Medical Science; 10(3-4): 85-92.

2. Agate L, Mariotti S, Elisei R, Mossa P, Pacini F and Molinaro E (2008): Thyroid Autoantibodies and Thyroid Function in Subjects Exposed to Chernobyl Fallout during Childhood: Evidence for a Transient Radiation-Induced Elevation of Serum Thyroid Antibodies without an Increase in Thyroid Autoimmune Disease. J Clin Endocrinol Metab; 93(7):2729-36.

3. Ai J, Leonhardt M and Heymann R (2003): Autoimmune thyroid diseases. Etiology, Pathogenesis, and dermatologic manifestations. J Am Acad Dermatol; 48:641-59.

4. Al-Juburi S, Taresh H, Mahmood A and AlFatlawi R (2015): The relationship between anti-thyroidal peroxidase antibodies and thyroid hormones (T3, T4 and thyroid stimulating hormone TSH) among patients with autoimmune thyroid disease. Europ Scient J; 3: 1857 - 1881.

5. Balazs C (2012): The role of hereditary and environmental factors in autoimmune thyroid diseases. Orv Hetil; 153 (26): 1013-22.

6. Biondi B and Cooper DS (2008): The clinical significance of subclinical thyroid dysfunction. Endocr Rev; 29(1): 76-131.

7. Bufalo NE, de Nadai AC, Barbieri RB, Marcello MA, Cunha LL, Morari EC and Ward LS (2011). Thyroid Disruptors: How They Act and How We React. In: Basic and Clinical Endocrinology Up-to-Date, Akin F (ed.), ISBN: 978-953-307340-8, InTech. pp. 6-7 http://www. intechopen.com/books/basic-and-clinicalendocrinology-up-to-date/thyroid-disruptorshow-they-act-and-how-we-react

8. Cardiso C, Maraes C, Teixeria J et al. (2014): Pattern of radiation exposure in health care professionals during Coronary Angiography. Rev Bras Cardiol Invasiva; 22(4):320-323.

9. Chida K, Kaga Y,Haga Y,Kataoka N, Kumasaka E, Meguro T et al. (2013): Occupational dose in interventional radiology procedures. AJR Am J Roentgenol ; 200:138-41.

10. Cousins C, Miller DL, Bernardi G et al. (2013): ICRP Publication 120: Radiological protection in cardiology. Ann ICRP; 42:1-125.

11. Eheman C, Garbe P and Tuttle R (2003): Autoimmune thyroid disease associated with environmental thyroidal irradiation. Thyroid; 13(5):453-64.

12. Elzaki A, Osman H and Lawz O (2012): Thyroid Nodules Development among Radiographers. J Adv Med Res; 2 (2): 79-89.

13. Ghoraishian S, Moghaddam S and AfkhamiArdekani M (2006): Relationship between Anti-Thyroid Peroxidase Antibody and Thyroid Function Test. Iran J Immunol; 3 (3):146-9.

14. Goodnow CC (2001): Pathways for selftolerance and the treatment of autoimmune diseases. Lancet; 357:2115-21.

15. Hamatani $K$, Eguchi $H$, Ito $R$, Mukai $M$, Takahashi K, Taga M, et al. (2008): RET/ PTC rearrangements preferentially occurred in papillary thyroid cancer among atomic bomb 
survivors exposed to high radiation dose. Cancer Res; 68 (17): 7176-82.

16. Hawa M, Picardi A, Costanza F, D'avola D, Berreta G, Anguissola Net al .(2006): Frequency of diabetes and thyroid auto-antibodies in patients with autoimmune endocrine disease from Cameroon. Clinic Immunol; 118(2-3): 229-32.

17. Kemp E (2004): Auto-antibodies as diagnostic and predictive markers of vitiligo. Autoimmunity; 37:287-90.

18. Kim K, Miller D, Balter S, Kleinerman R, Linet M, Kwon D et al. (2008): Occupational radiation doses to operators performing cardiac catheterization procedures. Health Physics; 94(3): 211-27.

19. Kindler S, Roser M, Below H, Hoffmann W, Kohlmann T, Kramer A et al., (2006): Thyroid disorders in employees of a nuclear power plant. Thyroid; 16(10): 1009-17.

20. Mettler F, Bhargavan M, Faulkner K, Gilley D, Gray J, Ibbott G et al., (2009): Radiologic and nuclear medicine studies in the United States and worldwide:frequency, radiation dose, and comparison with other radiation sources--1950-2007. Radiology; 253(2):52031.

21. Nizovtsova L and Mozhzhukhina I (2005): Comparative results of thyroid ultrasound and laboratory studies in patients exposed to radiation. Vestn Rentgenol Radiol; (6):20-6.

22. Ostroumova E, Brenner A, Oliynyk V, McConnell R, Robbins $\mathrm{J}$ and Terekhova $\mathrm{G}$ (2009): Subclinical hypothyroidism after radioiodine exposure: Ukrainian-American cohort study of thyroid cancer and other thyroid diseases after the Chernobyl accident (19982000). Envi Health Persp; 117 (5):745-50.

23. Rehani M and Ortiz-Lopez P (2006): Radiation effects in fluoroscopically guided cardiac interventions, keeping them under control. Int J Cardiol; 109 (2):147-51.

24. Ron E and Brenner A (2010): Non-malignant thyroid diseases after a wide range of radiation exposures. Radiat Res; 174:877-88.

25. Völzke H, Werner A, Wallaschofski H, Friedrich N, Robinson DM, Kindler S et al. (2005): Occupational Exposure to Ionizing Radiation Is Associated with Autoimmune Thyroid Disease J Clin Endocrinol Metab; 90(8):4587-92.

26. Walsh J, Bremner A and Bulsara M (2005): Parity and the risk of autoimmune thyroid disease. A community-based study. J Clin Endocrinol Metabol; 90(9): 5309-12.

27. Yoshimoto Y, Ezaki H, Etoh R, Hiraoka T and Akiba S (1995): Prevalence rate of thyroid diseases among autopsy cases of the atomic bomb survivors in Hiroshima, 1951-1985. Radiation Research; 141: 278-86. 